\section{Mittelohrimplantate auch für Kinder mit Gehörgangsatresie}

$B_{k}^{e}$ ei Innenohr-, Schallleitungs- und kombinierter Schwerhörigkeit gewinnen Mittelohrimplantate zunehmend an Bedeutung, berichtete Professor Dr. Stefan Dazert, Direktor der HNO-Klinik des St. Elisabeth-Hospitals Bochum. Damit können beispielsweise auch Kinder mit einer Gehörgangsatresie, das heißt einer sogenannten großen Ohrfehlbildung ohne Gehörgangsanlage, versorgt werden.

Mittelohrimplantate wie die Vibrant Soundbridge erlauben unter Erhalt der Anatomie eine variable Anpassung an
Mittelohrstrukturen und ermöglichen eine direkte und präzise Signalübertragung auf die Gehörknöchelchen oder die Fenster zum Innenohr. Durch die direkte mechanische Stimulation von Mittelohrstrukturen wird die Klangqualität gegenüber einem Hörgerät deutlich verbessert.

Eine neue Entwicklung stellen Knochenleitungsimplantate dar, die bisher allerdings noch nicht für das Kindesalter zugelassen sind. Das Bonebridge-System besteht aus einem Implantat, das komplett unter der Haut liegt und im Felsen- bein, z.B. im Sinus-Durawinkel, verankert wird, sowie einem extern getragenen Audioprozessor, der durch einen Magneten am Implantat gehalten wird. Das Implantat wandelt empfangene Signale in mechanische Schwingungen um, die über den Knochen direkt an das Innenohr übertragen und von dort an den Hörnerv weitergeleitet werden.

Erste Erfahrungen sind vielversprechend, berichtete Dazert. Komplikationen wie gehäufte Mittelohrinfektionen oder Probleme infolge des Wachstums des Kindes seien voraussichtlich nicht zu befürchten, Langzeitergebnisse bleiben aber abzuwarten.

Dazert S. Implantierbare Hörsysteme bei Kindern.

\title{
Stottern: Frühzeitige Behandlung statt langes Abwarten
}

S orgen, weil ein Kind stottert, werden häufig beschwichtigt: Stottern im Kindesalter weist eine hohe Spontanheilungsrate von 70 bis 80 Prozent auf. Bleibt das Stottern jedoch bestehen, wirkt es sich nachweislich negativ auf soziale Beziehungen, Ausbildung und Berufsleben aus, warnte Professor Dr. Karin Neumann, Abteilung für Phoniatrie und Pädaudiologie der Universität Bochum. Nach der Pubertät wird eine Therapie schwierig. Im Vorschulalter dagegen sind die Heilungschancen sehr gut. Neumann plädierte daher für eine frühzeitige Therapie, mit der sich hohe Komplettheilungsraten erzielen lassen, die deutlich über den Effekt der Spontanremission hinaus gehen.

Wie die Versorgungsrealität zeigt, wird jedoch häufig eine Waitand-see-Strategie verfolgt oder die verordneten Therapien sind wenig effektiv, kritisierte Neumann. Ihre Arbeitsgruppe untersuchte in einer retrospektiven Befragung von 88 Patienten, wie effektiv verschiedene Interventionsformen waren. Die Patienten hatten sich im Durchschnitt drei Therapien unterzogen. Eine unspezifische logopädische Behandlung, die im Kindesalter die häufigste und am längsten andauernde Ersttherapie darstellt, zeigte ebenso wie die Atemtherapie und die Hypnose eine unbefriedigende Wirkung. Die Effektivität der Fluency-shaping-Therapie und der Stottermodifikation war dagegen gut. Zu den Fluency-shapingTherapien, die auf eine Verbesserung der Sprechflüssigkeit abzielen, zählt beispielsweise die Kasseler Stottertherapie. Eine Stottermodifikation wie KIDS („Kinder dürfen stottern“) legt den Schwerpunkt auf den Abbau von psychischen Belastungen durch das Stottern und soll das Selbstbewusstsein des Kindes stärken.

Ein weiterer Ansatz, der in Deutschland noch neu ist und daher nicht in die Studie einbezogen werden konnte, ist das LidcombeKonzept zur Frühtherapie kindlichen Stotterns im Vorschulalter. Dabei werden die Eltern als Co-Therapeuten einbezogen und darin unterwiesen, täglich in strukturierten und unstrukturierten Spielsituationen flüssige Äußerungen des Kindes zu loben und durch positive Rückmeldung zu verstärken. Bei Stotterepisoden wird das Kind - angepasst an die individuellen Fähigkeiten - be- hutsam zu einer Korrektur angehalten. Nach und nach erfolgt die Stabilisierung der Sprechflüssigkeit durch positive Verstärkung in zeitlich zunehmend größeren Abständen. Damit lasse sich nicht nur die Stottersymptomatik signifikant und ohne Rückfallgefahr reduzieren, sondern auch die oft belastete Eltern-Kind-Interaktion entlasten, sagte Neumann. Wesentlich sei eine Verlaufskontrolle und individuelle Anpassung durch einen geschulten Therapeuten.

Neumann empfahl, bei Auftreten einer Stottersymptomatik nicht länger als sechs Monate zu warten. Dann sollte das Kind statt einer wenig wirksamen unspezifischen Logopädie eine der vorgestellten evidenzbasierten Stottertherapie bei einem spezialisierten, zertifizierten Therapeuten erhalten.

Neumann K. Stottern - was tun?

Berichterstattung: Angelika Bauer-Delto

Anzeige

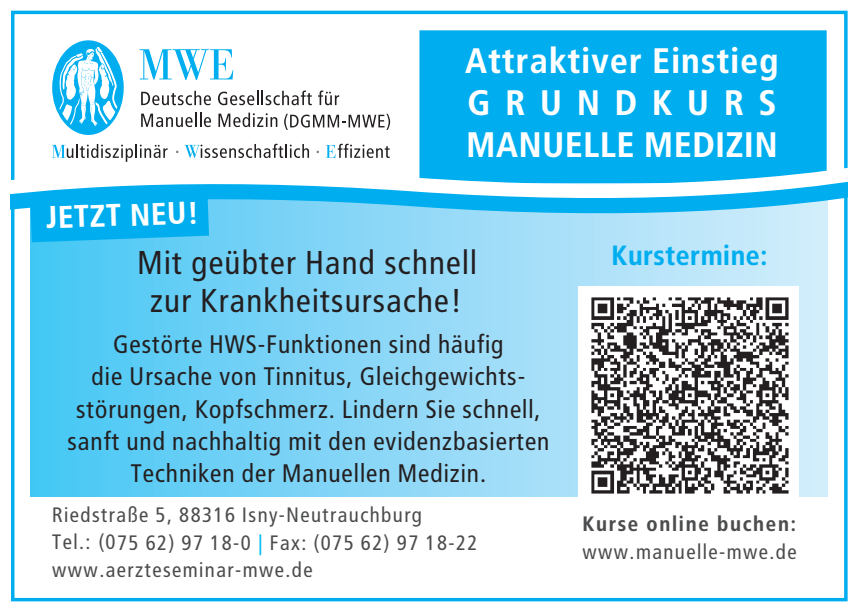

